\title{
Validation of a coupled pressure-equalization-thermal- mechanical model to study double-skin facades
}

\author{
Nathan Van Den Bossche ${ }^{1, *}$, Kjartan Van den Brande ${ }^{1}$, Henk De Bleecker $^{2}$ and Guido Lori ${ }^{2}$ \\ ${ }^{1}$ Ghent University, Faculty of Engineering and Architecture, Sint Pietersnieuwstraat 41 B4, 9000 Ghent, Belgium \\ ${ }^{2}$ Permasteelisa Group, Group Innovation and Technology, viale Mattei 21/23, 31029 Vittorio Veneto, Italy
}

\begin{abstract}
Although double skin façades are a well-known façade system, still few experimental data and numerical approaches exist in literature that couple their thermal behaviour and pressure equalisation with the structural response. The reason is that, differently from insulating glazing units, the extent of the problem is trivial when designing ventilated double skins. Indeed, the leakage of the double skin cavity is large enough to avoid any response of the internal cavity pressure to its temperature variations. However, during the last years Closed Cavity Façades, a new trend of double skins, have been applied in order to achieve high performances in terms of thermal and acoustic insulation and providing at the same time valuable benefit with regards to maintenance cost reduction. The CCF low levels of air permeability are responsible for a strong correlation of pressure and temperature of its cavity and the phenomenon needs an accurate model to support the design. Indeed, an optimal design of the outer and inner skin should consider the skin interactions and their coupling through the cavity, under the dynamic temperature and variable mass within the cavity itself. The mass variation is governed by two counteracting effects: on one side the dry and clean air that is pumped into the cavity in order to avoid the risk of condensation, on the other side the mass flows through the skin openings that connects cavity with external and internal environment. Moreover, the pressure is also affected by the interior and exterior air pressures that induce a deflection of the inner and outer skin. A numerical calculation procedure was developed, integrated with commercial codes commonly used in façade design, in order to provide a unique assessment tool for the coupled thermal-mechanical design of double skin facades. The tool has been validated by means of an extensive experimental campaign and the different relevant effects have been described.
\end{abstract}

\section{Introduction}

Double skin facades are a well-known façade system that has been studied in detail since several years, especially in term of its thermal insulation efficiency [1-3]. However, a lack of experimental data in the correlation between its cavity temperature and the structural actions on the skins can be noticed. Obviously the problem has not been investigated in detail as it is not relevant for the most frequent design of double skin facades, characterized by a highly ventilated cavity. Looking at the most critical ventilated double skins in terms of cavity temperature conditions (like for instance the spandrel areas of curtain wall facades), it is common to have geometrical conditions for the opening, which results in more than $0.1 \%$ of relative permeability, limit stated by the codes to define a skin impermeable. Under these conditions, the mass flow due to the pressure-equalization mechanism is sufficient to balance the temperature impact in terms of cavity volume expansion, with a net effect of no pressure raise within the cavity [4-8] implicitly adopt wind pressure as primary load for double skin façades). However, it is clear that systems characterized by lower levels of permeability could be affected by relevant mechanical actions. For this reason, the thermalmechanical coupling is a fundamental topic of the double skin design.

\subsection{Insulating glazing units versus double skins}

The thermal-mechanical behaviour of a cavity can be described by means of the gas state equation:

$$
P V=m R T
$$

In which $\mathrm{P}$ : pressure [Pa], V: volume $\left[\mathrm{m}^{3}\right], \mathrm{m}$ : number of moles [-], R: ideal gas constant [J/mol.K], T: temperature $[\mathrm{K}]$. In case of variable temperature over the cavity volume, $\mathrm{T}$ represents the average temperature. By expressing the pressure as a function of the other two state variables and differentiating versus time, we obtain:

$$
\frac{d P}{d t}=\frac{m R}{V}\left(\frac{d T}{d t}-\frac{T}{V} \frac{d V}{d t}\right)
$$

Equation (2) describes the rate of the pressure increase when a temperature rate is applied into a closed cavity. In case of infinitely rigid skins, it is simplified in a linear

\footnotetext{
* Corresponding author: Nathan.VanDenBossche@ugent.be
} 
relationship, driven by the slope $m R / V$. More realistic conditions of deformable skins give lower pressures as a function of skin geometry and stiffness. When equation (2) is extended to open cavity conditions, the mass also becomes variable, adding a third term to the equation:

$$
\frac{d P}{d t}=\frac{R}{V}\left(\frac{T d m}{d t}+m \frac{d T}{d t}-m \frac{T}{V} \frac{d V}{d t}\right)
$$

The equation (3) is now taking in account that the mass can change and this effect balances the potential raise due to the temperature rate, until the mass flow is reasonably greater. In general, the mass flow is driven by the pressure-equalization principle, which says that the mass flow Q through an opening of size A, depends on the pressure difference $P$ between the two environments connected by the opening and by the density of the air according to the equation:

$$
Q=\frac{d m}{d t}=c_{D} A_{c a v}\left(\frac{2 \Delta P}{\rho}\right)^{\left(\frac{1}{b}\right)}
$$

Where $b$ is the flow exponent and $c_{D}$ the discharge coefficient. It is clear that as a function of the cavity permeability $A_{\text {cav }}$, the pressure build-up in the cavity goes from the maximum rate expressed by (2), when the permeability is zero, to zero, when the permeability is large. For façade units with a high airtightness it is impossible to explicitly measure the area of the "gaps" $A_{\text {cav }}$ and therefore also impossible to measure the discharge coefficient $C_{d}$. The permeability of the cavity, mentioned in this paper, incorporates the product of these two parameters. Also, in this research the flow through these "gaps" is assumed to be turbulent $(b=0.5)$.

\subsection{Closed Cavity Façades}

During the last years a novel type of double skin facade has been applied more frequently, especially under high demand for visual appearance and maintenance cost reduction. The closed cavity is a double skin characterized by high airtightness (low permeability) and mechanically supplied by a continuous dry air flow in the cavity [9]. In this way the ingress of dust is minimised and the relative humidity is mitigated, lowering the risk of condensation. For these reasons, the understanding of its thermalstructural coupled behaviour is a fundamental objective, in order to provide a sustainable design for its skin components.

In this paper, a pressure-equalization thermalmechanical model will be presented and validated, in particular making use of experimental measurements conducted on a CCF façade element, subjected to the superimposition of the solar radiation and dry air flow effects.

\section{A simple numerical model to analyse double skin facades}

A numerical model has been developed and validated [10] and extended in terms of computational capabilities by
Permasteelisa Innovation and Technology Group, in order to provide an efficient simulation of the structural behaviour of the close cavity façade under the different structural actions during its serviceability life.

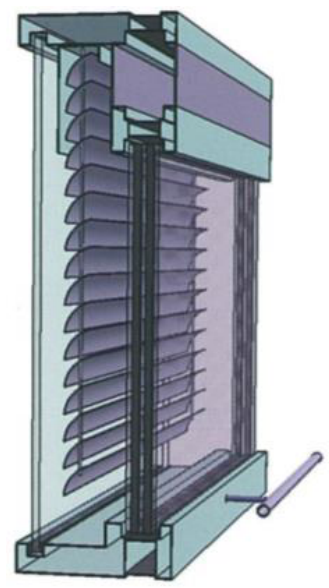

Fig. 1. Example of unitized mfree-S CCF façade (moisturemaintenance free Sustainable Close Cavity Façade)

The model may be regarded as an extension of the wellknown insulating glazing unit model largely adopted nowadays for the design of facades. The key principle for the solution of the structural system consists of the pressure equilibrium between the volume change undergone by the gas and the variation of the volume of the skins under interior and exterior pressures. The only significant difference is represented by the possibility to accomplish the two major differences between an insulating glazing unit and a closed cavity:

- Dry air flow continuously pumped into the cavity, in order to control the $\mathrm{RH}$ and avoid risk of condensation

- The skin permeability towards internal and external of the building, which is a fundamental design parameter to make sure that the intended ventilation is established into the cavity

Both the items have a significant effect on the pressure conditions of the cavity and for the specific case of the closed cavity they determine a unique structural behavior, intermediate between the well-known conditions of pressure equalized cavity and closed insulating units. With reference to Fig. 2, we will make use of the classical layout for a mfree-S CCF facade, constituted by a vision area and a spandrel area separated by frame intermediate transoms. In order to capture the correct stiffness behavior of the inner skin double glazing unit, a two degrees of freedom numerical model will be used for the test-case of this paper, assuming no permeability effects through the DGU cavity $\left(A_{12}=A_{2 R S}=A_{2 A B}=0\right)$. The equilibrium system of equations can be written as:

$$
\begin{aligned}
& \mathrm{Q}_{1}+A_{1 R S} \sqrt{\frac{2\left(P_{e x t}-P_{c 1}\right)}{\rho}}+A_{1 A B} \sqrt{\frac{2\left(P_{c 1}\right)}{\rho}}+A_{12} \sqrt{\frac{2\left(P_{c 1}-P_{c 2}\right)}{\rho}}=0 \\
& \mathrm{Q}_{2}+A_{2 R S} \sqrt{\frac{2\left(P_{e x t}-P_{c 2}\right)}{\rho}}+A_{2 A B} \sqrt{\frac{2\left(P_{c 2}\right)}{\rho}}-A_{12} \sqrt{\frac{2\left(P_{c 1}-P_{c 2}\right)}{\rho}}=0
\end{aligned}
$$

The equations use the classical notations for flows $Q_{i}$ and for the $A_{i R S}$ (rainscreen, to the external) and $A_{i A B}$ (air 
barrier, to the internal) equivalent areas of permeability, derived by the rainscreen theory. In addition, the mutual cavity $A_{12}$ area is defined. Discharge coefficient have been considered incorporated into the equivalent opening permeability. Under rigid skin conditions and in stationary equilibrium for general skin behavior, equations (5) and (6) are sufficient to determine the equilibrium of the system. When the skins are deformable, (5) and (6) should be considered in incremental form and must be coupled with the equilibrium pressure of the single cavity, where the coupling is through the variable mass contribution $m_{i}$, given by the application of (1) and (2) within the time interval:

$d V_{g a s}\left(P_{c i}, T_{c i}, m_{i}\right)-\sum d V_{\text {skin }}\left(P_{\text {netskin }}\right)=0$

Where the elemental volume gas variation should be assessed on the basis of the variation of the pressure and cavity average temperature with respect to the reference conditions and the skin volume variation will be the lump sum of the volume changes with respect to the undeformed condition of all the surfaces of all the skins constituting the boundary of the cavity.
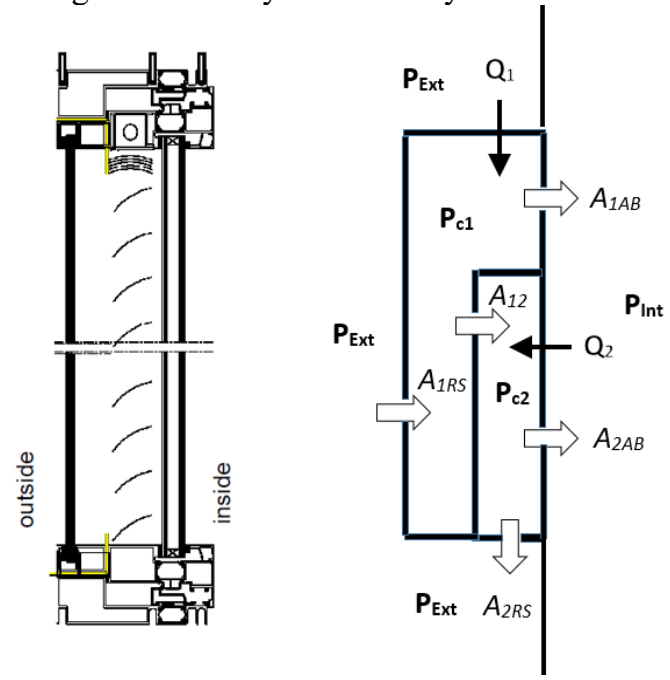

Fig. 2. Pressure equalization-mechanical model of a CCF

One of the major advantages of the described tool is that it is coupled with a FEM commercial code, in order to generate the shape functions of the single skin surface under the net pressures. Once a certain geometry has been selected, the required surface "shape functions", like displacement versus pressure, volume versus pressure and maximum stress versus pressure function are generated or accessed from a database, used to store already calculated "shape functions". In case of laminated glass, the required Young Module of the interlayer can be selected. The other inputs required for the run of the tool are the permeability values (or functions) of the single skins, which will be discussed more in details during the next paragraphs and the time histories of wind, cavity temperature and flow. Running a Newton-Raphson solver of the multiple equation system, the primary output of the calculation will consist of the pressure time history of the single cavities, but by means of the above mentioned "shape functions" of each surface, the time histories of displacement and maximum stress under the specific net pressures can also be derived.

\section{Experimental test and model validation}

During the period June-December 2019, a façade panel of a closed cavity façade has been instrumented in order to capture the thermal-mechanical response of the panel under different environmental conditions. The panel has been integrated in a box, like shown in Fig.3, with the façade exposed to the south. The readings have been analysed and used in order to calibrate the numerical model proposed for the study of the thermal-structural coupling behaviour of double skin façades.

\subsection{Experimental set-up}

The façade unit has dimensions of $3 \mathrm{~m}$ in width and $4 \mathrm{~m}$ in height. It is composed of an outer skin and an inner skin divided in 2 surface areas by an intermediate transom. Bottom surfaces are glazed, whereas top spandrel are metal panels. The glazed area is $3.5 \mathrm{~m}$ in height and it is composed of a single laminated glass of 2 glass layers with $5 \mathrm{~mm}$ annealed glass each and $0.76 \mathrm{~mm}$ PVB interlayer. Inner vision is a double glazing unit with a $6 \mathrm{~mm}$ monolithic annealed glass, $16 \mathrm{~mm}$ air space and still a $2 \times 5 \mathrm{~mm}$ annealed glass with $0.76 \mathrm{~mm}$ PVB interlayer. Cavity depth is about $215 \mathrm{~mm}$. The climatic test-box has been undergoing variations of environmental (solar radiation/wind) and imposed conditions (dry air flow, blind position and orientation). In particular, the dry air flow has been varied in the range $100-3001 / \mathrm{h}$, in order to evaluate the pressure build-up response. The blind positions have been changed from position up, position mid-height, and position down. Blind slat orientation has been changed from $0^{\circ}$ (open) to $90^{\circ}$ (closed), with intermediate $30^{\circ}$ and $60^{\circ}$. A wide range of instruments have been adopted for the measurements of the major thermal-structural response of the unit: laser gauges have been used to measure the displacement of inner glass, outer glass and intermediate outer transom. Framing member deflections from the internal of the box have been recorded by LVDTs, while differential pressure sensors have been used to measure pressures in the cavity and acting on the external surface of the outer skin. Finally, an extensive set of thermocouples has been adopted to record the most relevant temperatures of frame, glass, blind, plates and air on each side of the cavity and in the cavity.

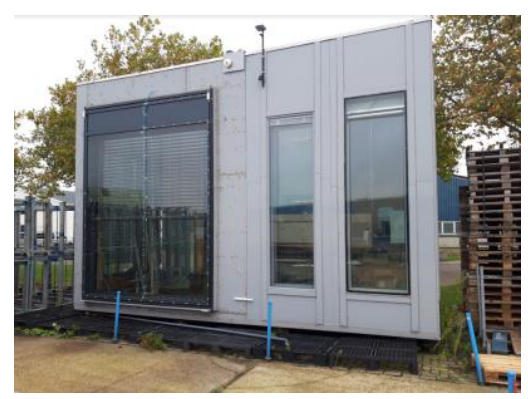

Fig. 3. Climatic Test-box with instrumented panel 


\subsection{Analysis of the data}

The analysis of the collected data has allowed to find several important behaviours of the closed cavity façade unit as a function of its permeability level and mechanical properties. Fig. 4 shows the time histories of the cavity pressure during one week of October in comparison with the average value of the different air temperatures measured in the cavity.

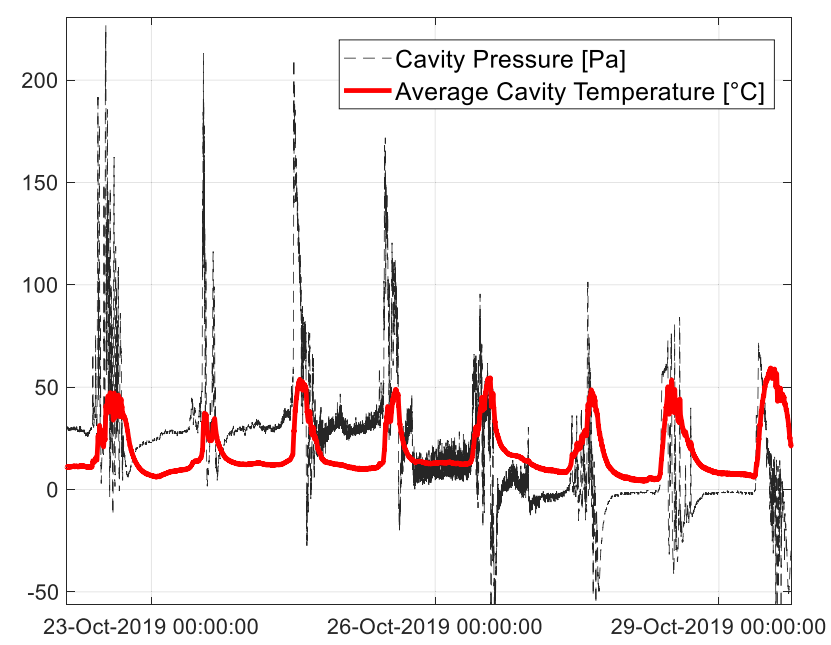

Fig. 4. Cavity pressure and average temperature time histories

The thermal cycles due to the daily solar radiation are apparent, with the consequent pressure raise in the cavity. It can also be seen that the "mean" pressure is typically not zero and this effect is due to the presence of the mechanically introduced air flow, which renders a pressure increase in the cavity, and due to the cavity permeability and wind pressure.

\subsubsection{Permeability function}

A second important finding is that the permeability of the closed cavity façade is function of the applied pressure due to geometric changes. The knowledge of this function is paramount for the application of the numerical tool and it can be considered as a preliminary input, which otherwise would be complex to extrapolate from all the other effects superimposed in the climatic test box measurements. The different permeabilities at different cavity pressures are derived from the equation for the calculation of the pressure under a certain constant flow $Q$ as described in equation 4 . As a first step of the procedure, the cavity pressure under a certain imposed airflow can be used to extrapolate towards a leakage rate as a function of pressure, as shown in Fig.5.

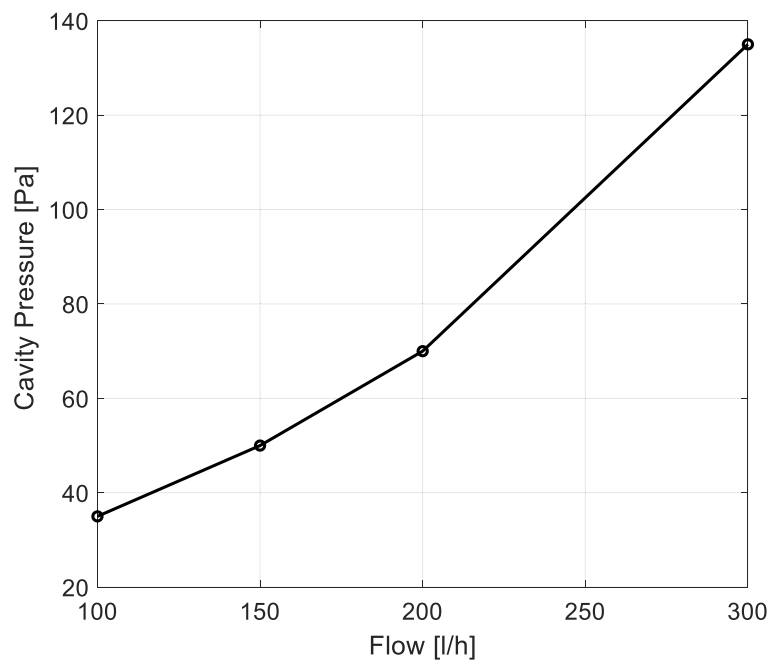

Fig. 5. Estimated function cavity pressure versus flow

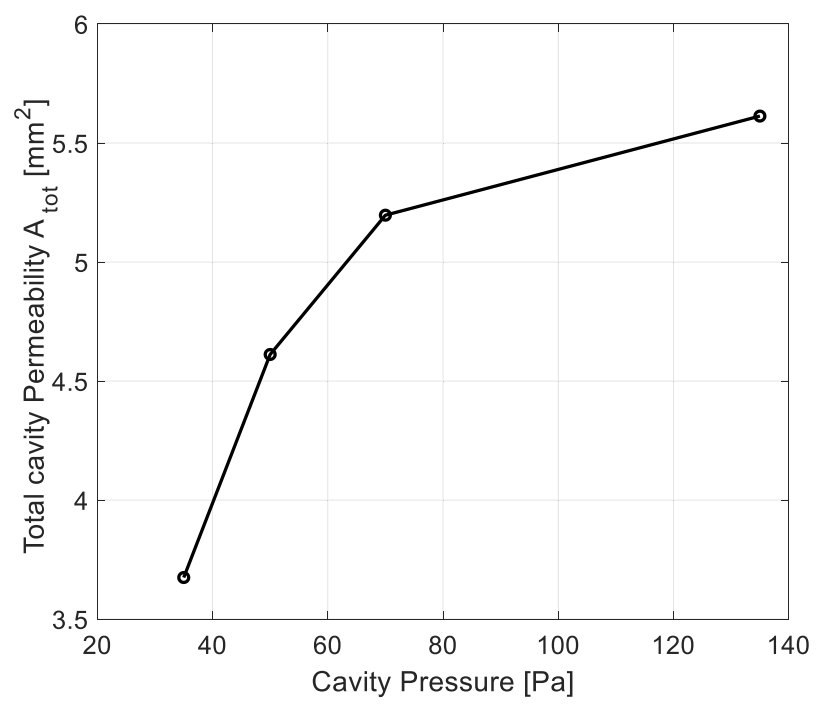

Fig. 6. Estimated function cavity permeability versus pressure

In a second step, equation (4) permits the estimation of the total cavity permeability $\mathrm{A}_{\text {Tot }}$ for the different combinations of pressure and flow, as shown in Fig.6. For this purpose, it is important to omit the effect of temperature variations on the cavity pressure and thus it is recommendable to analyse the pressure during the night time. Indeed, under these conditions, only wind gust can interact with the dry air flow in order to modify the pressure in the cavity.

\subsubsection{Pressure versus temperature cycles}

Another important façade characteristic that can be extracted from the data is the path $\mathrm{P}(\mathrm{T})$ and in particular the correlation between the rate of pressure increase versus the rate of temperature increase in the cavity. As it can be deducted by the equation (3), this function depends mainly on the facade mechanical properties and on its permeability. It is worth noting that, while looking at one day of measurements, it can be recognized that when the 
temperature increases, the slope of the pressure versus temperature plot is almost constant.

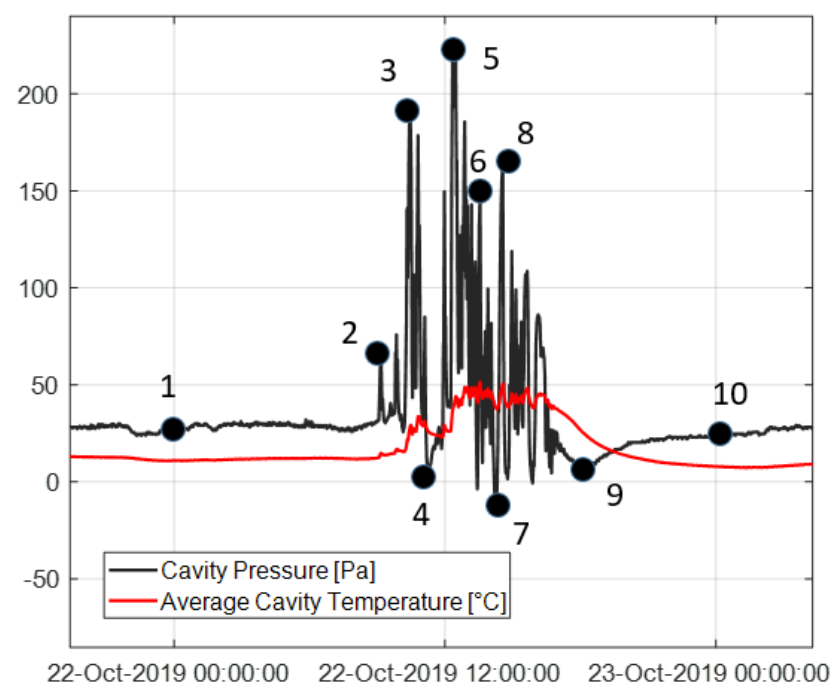

Fig. 7. Pressure build up and temperature raise time history in the cavity during the $22^{\text {nd }}$ of October

For instance, Fig.7 shows the time history of cavity pressure and average cavity temperature for the $22^{\text {nd }}$ of October, with 10 representative points highlighted. Point 1 is the starting of the day measurement and it occurs at midnight. Point 2 is the first daily pressure peak in the cavity at 9am, effect of the initial range of solar radiation. The day is characterized by clouds, which gives discontinuous period of air cavity heating. Point 3 corresponds to the higher peak of pressure during the morning at $10.30 \mathrm{am}$, followed by sudden pressure drops like point 4 (11.15am) that brings the cavity also to short periods of under-pressure with respect to the inner box pressure. Point 5 is the highest peak of pressure and it occurs at around 12.30. Still discontinuous moments of solar radiation characterize the afternoon, giving the highest temperature point 6 at $1.30 \mathrm{pm}$ and the lowest value point 7 of under-pressure at around $2.15 \mathrm{pm}$. Heating continues giving another cavity temperature peak at around 2.30pm (Point 8). It is interesting to note that the highest temperature time doesn't corresponds to the higher pressure in the cavity. The point 9 represents the relative pressure minimum at the end of the daylight and finally the point 10 is the midnight, beginning of $23^{\text {rd }}$ of October measurement. As discussed, it is interesting to show how the measurement of Fig.7 correlates in a pressure versus temperature plot, as shown in Fig.8. The plot highlights a likewise hysteretic behaviour with an almost constant slope that correlates the periods of temperature rate with the corresponding pressure rate, which is the characteristic thermo-mechanical coupling of the system, as expressed by equation (3). In similar way than for hysteresis cycles, period of "elastic" loading and unloading are connected by likewise plasticity periods, where the temperature changes without a significant change in pressure is appreciated, because of the slow variation. The major example is the cooling between point 9 and 10 , where the temperature goes from $40^{\circ} \mathrm{C}$ to $10^{\circ} \mathrm{C}$ without a change in pressure. On the contrary, intense loading and unloading pressure variations at similar temperatures occurs in between point 6 and point 9, characterized by very rapid changes of the temperature.

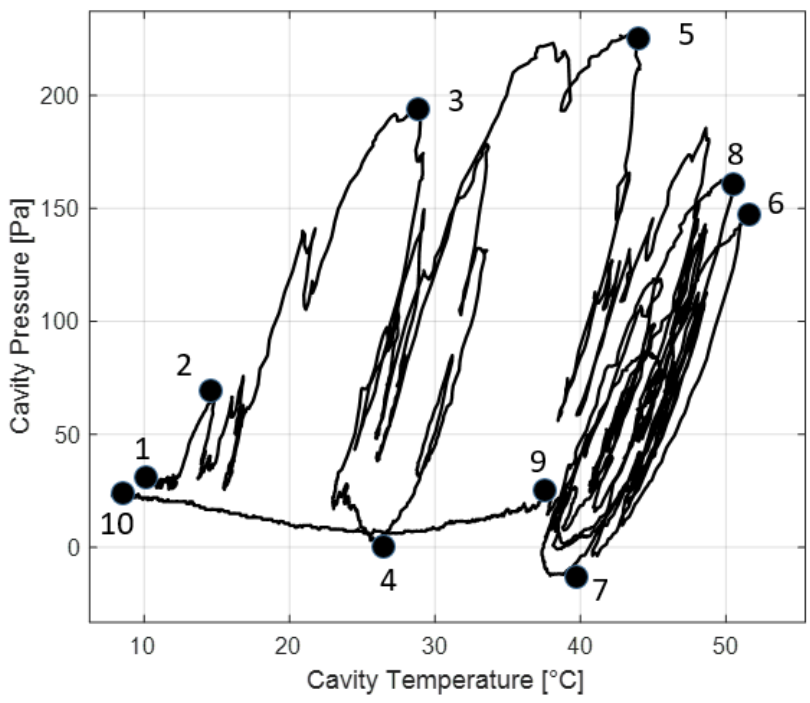

Fig. 8. Pressure build up in function of the temperature raise in the cavity during the $22^{\text {nd }}$ of October

\subsection{Numerical versus experimental}

In the previous paragraph the most important findings about the measurement analysis have been discussed. It has been noticed that the cavity total permeability is a fundamental parameter to describe the thermomechanical coupled behaviour of the cavity: in particular, permeability and structural parameters of the façade define how quickly the façade responds to the cavity temperature variations or, under another perspective, which speed of temperature variation becomes critical for the cavity, as its permeability and skin stiffness can't accommodate that rate of temperature without a sudden increase (or decrease) of the cavity pressure. At this scope it is important to have available for design purposes an accurate yet simple tool capable to predict the peaks of pressure against the combination of flow and cavity temperature variation. In this chapter, some examples of tool validation against the experimental measurements will be shown. Three scenarios have been selected in order to decouple the effects, according to the following descriptions.

\subsubsection{Scenario 1: Thermal cycle without flow / Low temperature}

The first scenario consists of a measurement during a day without mechanically introduced dry air flow and characterized by a small temperature raise in the cavity, mostly related to a cloudy day. The comparison between the tool estimation and the experimental measurement is shown in Fig.9. The fact that the temperature variation is small, allows to check the accuracy of the calculation based on the permeability estimated as explained at the previous step, because there is not much influence of the temperature on the laminated glass composite action. 
However, the low levels of pressure involved renders it important to properly evaluate the presence of wind pressure. For this reason, average pressure measurements on the external surface have been used as input for the tool as well. In the same graph it can be observed that the error, calculated as percentage of the ratio between the difference numerical/experimental and the experimental itself, is quite large for some time steps of the calculation, exceeding in some case the $50 \%$ value. However, in Fig.10, which shows the correlation between the error and the corresponding measured pressure, is highlighted that big differences occurs at the lowest level of pressure, which are not of interest for design purposes. For this reason, pressures lower than $3 \mathrm{~Pa}$ have been excluded in this case from the error plot, being characterized by a large but irrelevant error (in line with measurement uncertainty of the pressure sensors). For similar reasons, in the calculation of the error the first 5000s of the time history have been ignored, because of the time required to smooth the initial peak of pressures derived from the instantaneous application of the temperature and wind time histories. However, perhaps a different method for the application of the initial conditions could have been adopted in the simulation initialisation procedure, but that part of time history is in any case not relevant in terms of temperature significance levels.
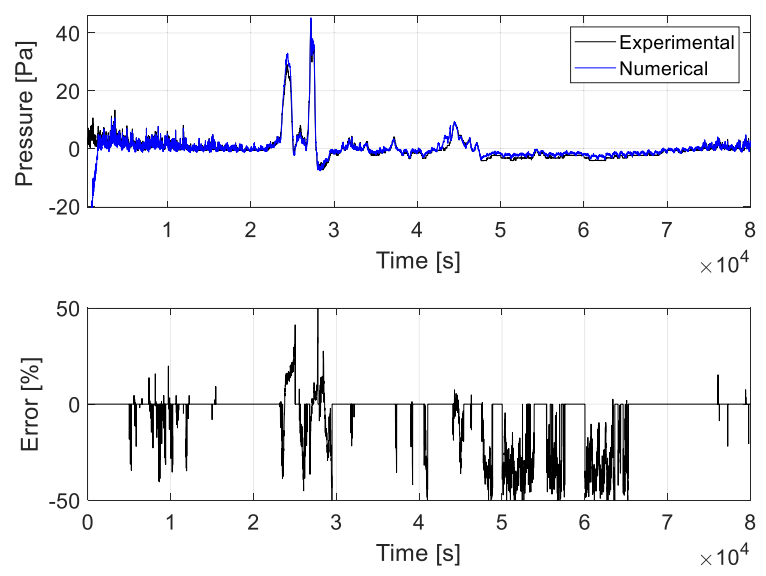

Fig. 9. Comparison experimental versus numerical and error for the cavity pressure measured during 3rd of November

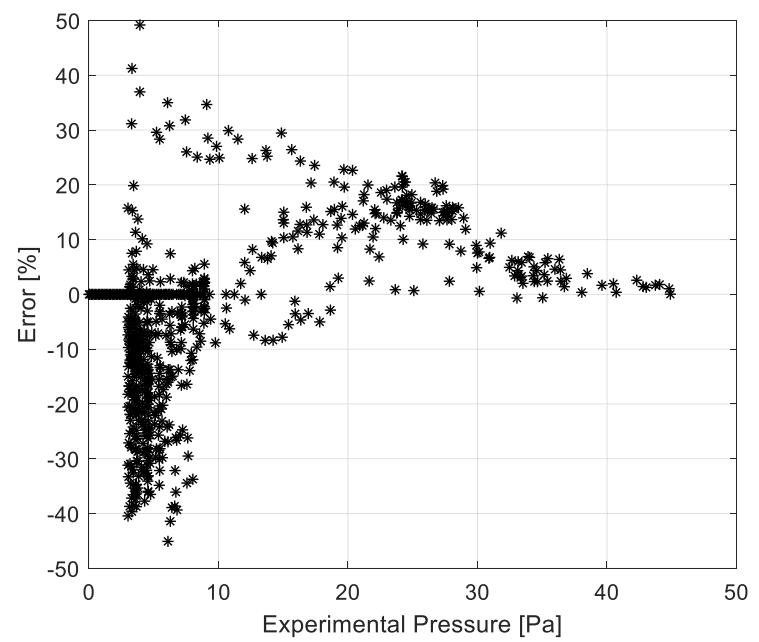

Fig. 10. Correlation between error and measure pressure during $3^{\text {rd }}$ of November

\subsubsection{Scenario 2: Thermal cycle without flow / High temperature}

Within this scenario it is possible to check the correspondence of the composite action of the laminated glass under different temperatures, and as well, the accuracy of the permeability function assumption on a larger pressure interval. An example of time history is shown in Fig.11. Also for this scenario it has been decided to ignore levels of pressure lower than a defined value (20Pa for this specific test) and due to initialisation of the simulation again the first 5000s of time history have been ignored. It can be observed in Fig. 12 that the error has a similar trend compared to the previous test case, being characterized by a smaller error for larger pressure differences. Under the environmental conditions of the $2^{\text {nd }}$ of November it can be seen that also relevant periods of negative pressures have been measured in the cavity. As for the previous case, the maximum error for peak values of design interest ranges in between 5 and $10 \%$.
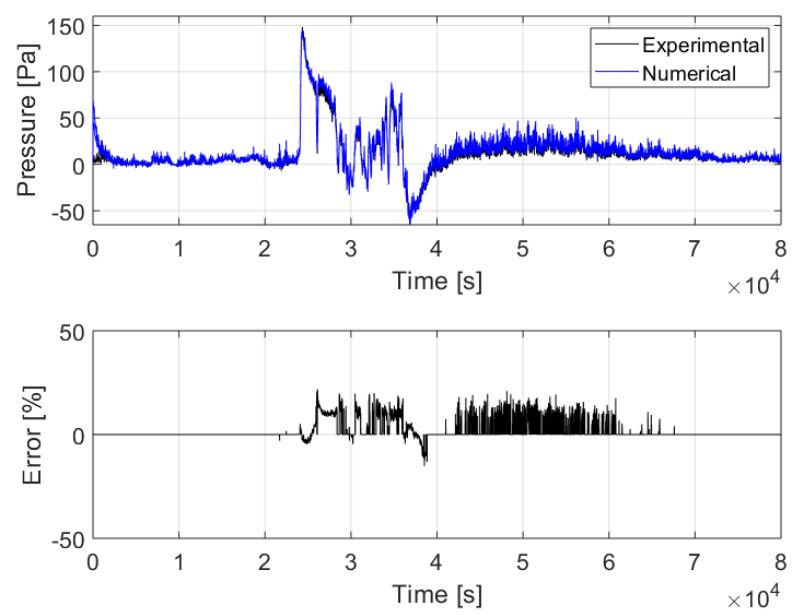

Fig. 11. Comparison experimental versus numerical and error for the cavity pressure measured during $2^{\text {nd }}$ of November

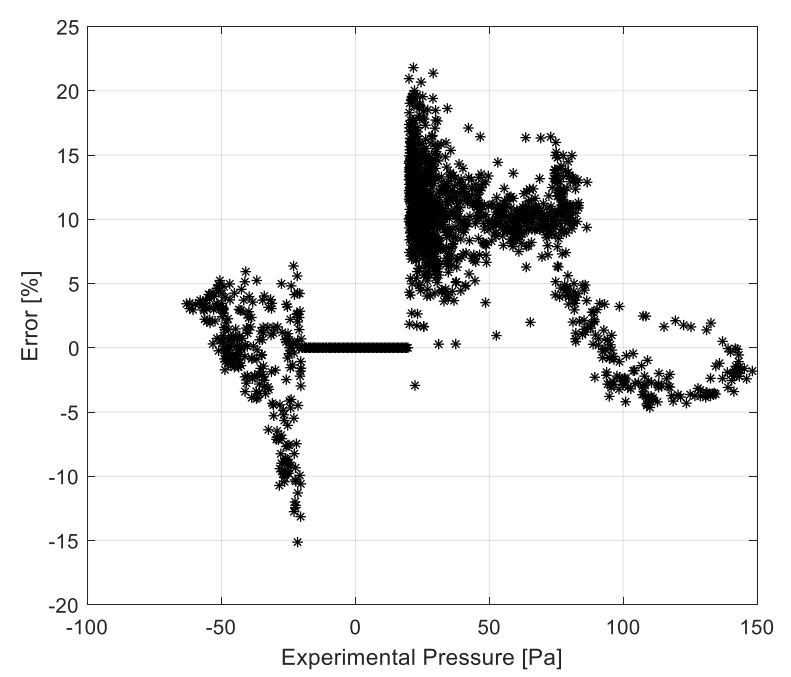

Fig. 12. Correlation between error and measure pressure during $2^{\text {rd }}$ of November 


\subsubsection{Scenario 3: influence of the dry air flow}

Finally, the superimposition of the flow with the temperature cycle in the pressure build up can be checked by considering a day with presence of flow and high solar radiation, like shown in Fig.13. It can be noticed from the analysis of the measurements that partially clouded days are more critical for the pressure build up, as they determine larger temperature speed rates and then larger pressure speed rates in the cavity, although in general they result in lower maximum temperatures in the cavity. Another confirmation from the measurements is that the pressure build up due to the flow cannot be considered as a simple linear combination with the effect of the temperature, as their superimposition is strongly nonlinear, as shown by equation 3 . The analysis of Fig. 13 and Fig. 14 confirms that the numerical tool seems adequate for the purpose of the assessment of the expected peak of pressure under a certain combination of cavity temperature, wind loading and dry air flow. Under the $22^{\text {nd }}$ of October environmental conditions, the pressure has reached values of about $230 \mathrm{~Pa}$, which for large glazing surfaces already induce a significant load, to be properly considered in the load combinations for the façade design. Furthermore, the authors are investigating the comparison between the above pressure levels and the pressures that would be found under similar temperature conditions in an insulating glazing unit, as the latter is frequently the adopted approximation hypothesis for design purposes. Although this is not the topic of this paper, it appears clear that the permeability of a CCF, even if small, has a significant beneficial effect on the pressure build-up and the equivalence with a IGU would yield very conservative results, with associated increasing glass thickness, in turn leading to increased financial and environmental costs.
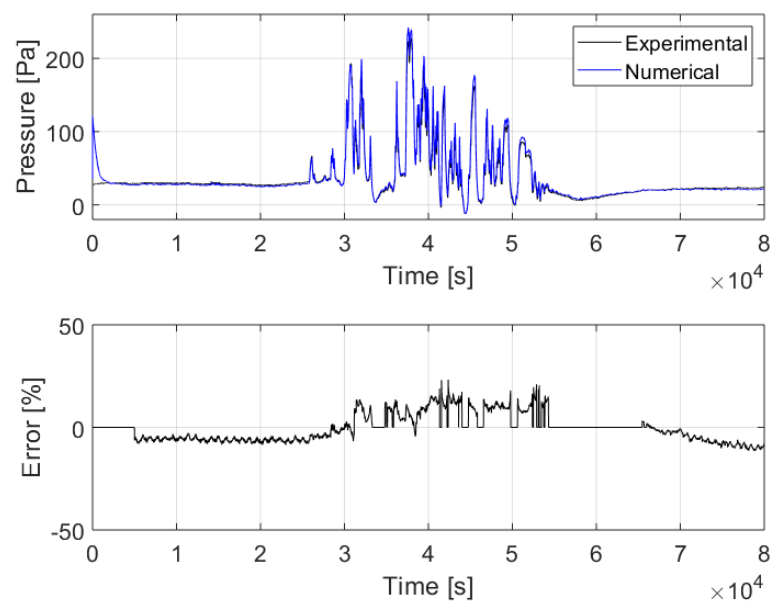

Fig. 13. Comparison of experimental versus numerical and error for the cavity pressure measured during $22^{\text {nd }}$ of October

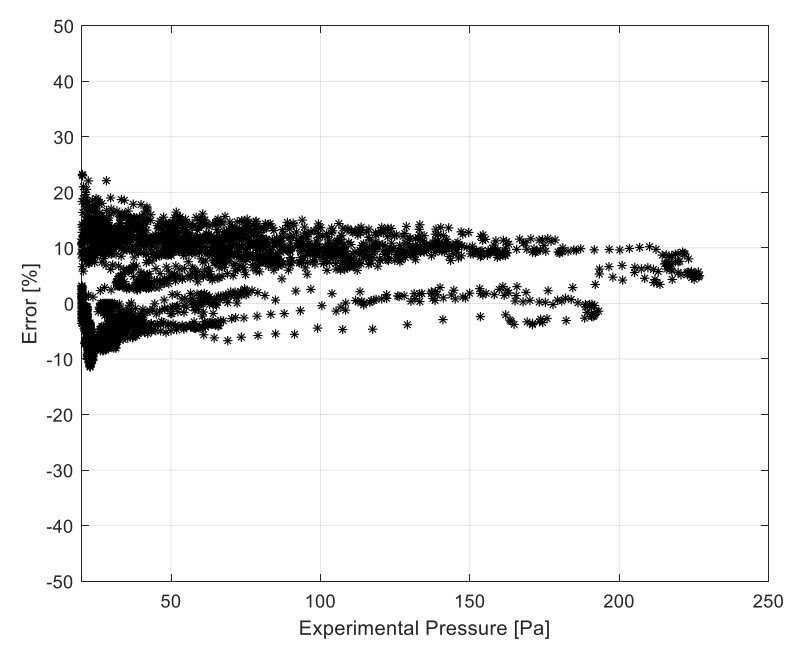

Fig. 14. Correlation between error and measure pressure during $22^{\text {nd }}$ of October

\subsubsection{Importance of the coupled pressure-equalized thermal-mechanical model}

The importance of a coupled pressure equalized thermalmechanical model to assess the cavity behaviour appears evident when the pressure build-up of the previous figures is compared with the outcomes of the tool, but making use of different hypothesis for the cavity stiffness and permeability. In Fig. 15, for instance, the coupled model outcome is compared with the assumption of nopermeability and with the permeable cavity but with rigid skins, under the cavity temperature conditions of the $2^{\text {nd }}$ of November. It can be noticed how in both the simplified scenario the pressure is significantly overestimated, with an average trend higher for the model without permeability, but with sharp peaks also for the model with rigid skins.

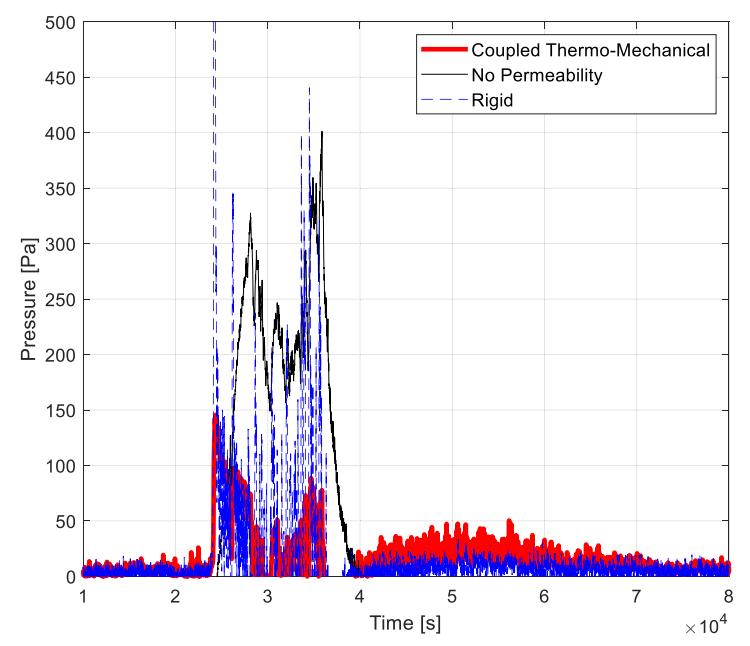

Fig. 15. Comparison between simulated pressure in the cavity under different assumptions for the cavity behaviour $\left(2^{\text {rd }}\right.$ of November) 


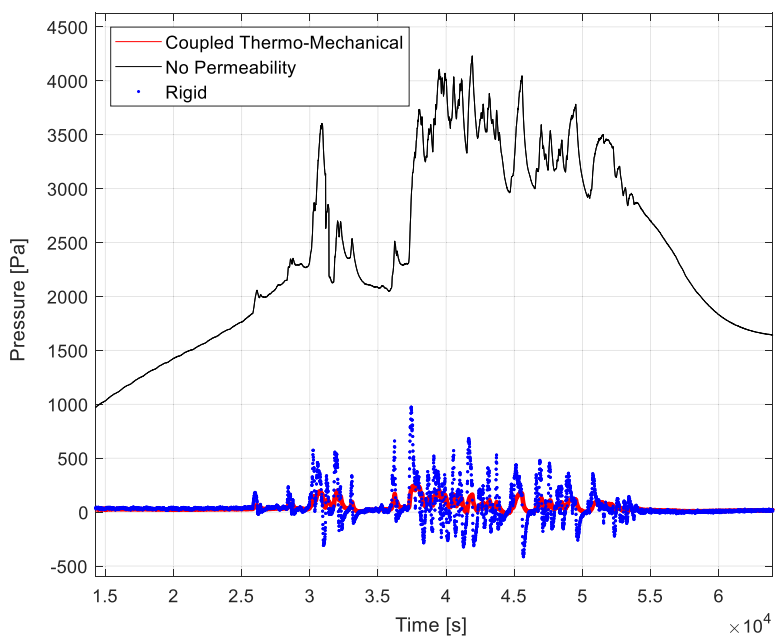

Fig. 16. Comparison between simulated pressure in the cavity under different assumptions for the cavity behaviour $\left(22^{\text {rd }}\right.$ of October)

Obviously the situation appears still more amplified when a day with dry flow presence is considered. Indeed, the calculation would be not actually possible, as the continuous flow would expand to the infinite the volume of the cavity. For this reason, a minimal permeability has been adopted for the cavity $\left(0.4 \mathrm{~mm}^{2}\right)$. Under these conditions the pressure at the flow stability would be of around $1.5 \mathrm{kPa}$ and under the temperature cycles more than $4 \mathrm{kPa}$ would be reached into the cavity. Even if the rigid hypothesis would be in this case less conservative than the no permeability assumption, still a factor around 5 is seen between the peak simulated by the pressure equalized thermo-mechanical model and the rigid skin scheme.

\section{Conclusions and future work}

The paper has been focused on the validation of a numerical tool for the assessment of the pressure build-up in the cavity of a CCF façade, under variable conditions of temperature and combination with other loads, like wind load and air flow effect. It has been demonstrated that the proposed tool seems adequate to capture the major trend in pressure variations and peak pressures, when calibrated on the basis of the permeability conditions of the sample and the mechanical properties of the façade. By dedicated comparisons, it has been also shown like a pressure-equalized thermo-mechanical model is required if the target is to estimate the pressure in the cavity in accurate way and without overly safe assumptions. Future work will be focused on further developments, which on the one hand would involve the integration of additional element deformability like effect of the framing members and the other hand would focus on a more extensive thermal model to account for all the interactions between the façade elements. In addition, the authors are continuing the validation and verification, comparing the experimental temperature time histories with the output of dedicated numerical tools. However, the tool seems already adequate, when provided with a robust sensitivity analysis of the major inputs, to be used for design purposes in order to support the design of double skin facades with low cavity permeability. Indeed, the major strength of this tool is the excellent compromise between accuracy and calculation time, as it runs one full day of simulation in few seconds. For this reason, the authors believe that it is worth to proceed the research and development work to still improve the performance towards the final objective of a full coupling with thermalsolar models that supplies the temperature time history in the cavity starting from the solar radiation and the thermal properties of the façade.

\section{References}

1. F. Pomponi, P.A.E. Piroozfar, R. Southall, P. Ashton, E.R.P. Farr, Energy performance of Double-Skin Façades in temperate climates: a systematic review and meta-analysis. Renew. Sustain. Energy Rev. 54, 1525-1536 (2016)

2. A. Ghaffarian Hoseini, A. Ghaffarian Hoseini, U . Berardi, J. Tookey, D.H.W. Li, S. Kariminia, Exploring the advantages and challenges of doubleskin facądes (DSFs). Renew. Sustain. Energy Rev. 60, 1052-1065 (2016)

3. H. Poizaris, Double skin facades for office buildings: literature review. Tech. Rep., Report EBD (2004)

4. Eurocode 1: Actions on Structures - Part 1:4: General Actions - Wind Actions

5. Luigi T. De Luca, Propulsion physics, EDP Sciences, Les Ulis, (2009)

6. H. Gerhardt, O. Kruger, Double skin glass facadesinvestigations into the load sharing possibilities. In: Proc. Of ICBEST, 97, 335-339 (1997)

7. F. Kemper, M. Feldmann, Wind load assumptions for permeable cladding elements considering the installation context. J. Wind Eng. Ind. Aerodyn. 184, 277-288 (2019)

8. F. Marques da Silva, M. Gloria Gomes, Gap inner pressures in multi-storey double skin facades. Energy Build. 40, 1553-1559 (2008)

9. J. Laverge, S. Schouwenaars, M. Steeman ,A. Janssens, N. Van Den Bossche, Condensation in a Closed Cavity Double Skin Facade: a Model for Risk Assessment. BEST Conference, Ottawa, Canada (2010)

10. N. Van Den Bossche, A. Janssens, M. Lacasse, T. Moore, The Impact of Pressure Equalization on Watertightness of Rainscreen Systems. International Building Physics Conference, Vienna, Proceedings. 783-790 (2013) 